Editorial

\title{
Importance of Quality Training in Neurology Speciality: Bangladesh Perspective
}

\author{
Md. Azharul Hoque \\ Professor \& Head, Department of Neurology, National Institute of Neurosciences \& Hospital, Dhaka, \\ Bangladesh; Email: editor_nins@yahoo.com
}

Medical education has undergone significant changes during the last decades. One of the reasons for the changes is the concern for patient's safety, which involves various cases of medical injury and medical malpractices those occurred around the world. Medical malpractice cases involve patients who have been harmed or injured due to poor medical treatment or mistaken diagnosis from a medical provider such as doctors, nurses, hospitals, other medical workers and medical device technicians.

Neurology is a medical specialty involving the management of conditions affecting the brain and other part of nervous system. The spectrum of patient groups in Neurology is incredibly broad and this is part of the appeal of the specialty. The specialty of Neurology is changing rapidly. Traditionally neurology had been thought of as an intellectual pursuit, concerned with diagnosis of rare conditions of the nervous system. The advent of accessible imaging, and the emergence of potential therapies, has led to neurologists concerned with the treatment and on-going care of disorders which are in fact very common such as stroke, epilepsy, multiple sclerosis, Parkinson's disease and dementia. Currently specialty training in neurology consists of five years in Bangladesh, one of which may be relevant research. Usually the training is based around regional neurosciences centres with rotation to other units. National Instituted of Neurosciences \& Hospital is the only referral neuroscience institute of Bangladesh.

One of the ways to enhance clinical competence is through Medical Simulation training. The training has been proven to have many advantages which help improve medical practitioners' competencies, and in return, improve patient safety and reduce health care costs. Medical simulation allows the acquisition of clinical skills through hands-on practice rather than an apprentice style of learning. With the recent advancement in technology, simulation tools serve as an alternative to real patients, which allow trainees to perform procedural mistakes and learn from them without the fear of harming the patient.

While hands-on learning with real patients cannot be completely replaced, simulation training provides a safe environment for learning. Making mistakes can be a valuable part of the learning process. In the simulation environment, patients are not put at risk. According to the Society for Simulation in Healthcare, simulation training is the imitation or representation of one act or system by another and serves as a bridge between classroom learning and real-life clinical experience. No longer must students practice give injections by using an orange or budding surgeons practice suturing by using a piece of felt. Today's patient simulators are significantly more advanced. Most of them can blink, breathe, cry, sweat, and have a heartbeat and pulse.

Simulation-based learning is expensive. There are different types and classification of simulators and their cost vary according to the degree of resemblance to reality, or 'fidelity'. However, this type of medical education is cost-effective if utilized properly.

Now more than ever, medical educators at all levels are being tasked with delivering foundational concepts and content necessary for the next generation of physicians and physician scientists to incorporate evidence-based medicine into their clinical practice and research. Physicians need the skills to evaluate the quality and relevance of content that they are incorporating to their expanding medical knowledge database. The emphasis of modern medical education should therefore focus on acquiring, interpreting, and applying new knowledge rather than committing old (and rapidly outdated) knowledge to memory.

[Journal of National Institute of Neurosciences Bangladesh, 2019;5(1): 1] 